\title{
'Dry' Pericarditis with Rapid Progression to Tamponade as a Feature of COVID-19
}

Authors:

Disclosure:

Received:

Accepted:

Keywords:

Citation:
*Ashwin Reddy, ${ }^{1}$ Sarah Nethercott, ${ }^{2}$ Rudolph Duehmke, ${ }^{3}$ Sunil Nair, Omar Abdul-Samad ${ }^{3}$

1. Royal Papworth NHS Foundation Trust, Cambridge Biomedical Campus, Cambridge, UK

2. Addenbrookes Hospital NHS Foundation Trust, Hills Road, Cambridge, UK

3. James Paget University Hospital NHS Foundation Trust, Lowestoft Road, Gorleston-on-Sea, Great Yarmouth, UK

*Correspondence to ashwin.reddy@cantab.net

The authors have declared no conflicts of interest.

08.10 .20

17.11 .20

Cardiac arrhythmias, cardiac imaging, coronavirus disease (COVID-19), pericardial disease, pericardiocentesis.

EMJ. 2021; DOI/10.33590/emj/20-00244

\section{Abstract}

Pericardial inflammation is a recognised feature of coronavirus disease (COVID-19). The authors herein present the case of a female with severe acute respiratory syndrome coronavirus-2 (SARS-CoV-2) infection who developed a large and life-threatening pericardial effusion over a few days following the onset of pericarditis, despite prompt commencement of treatment. This was successfully drained, and she was discharged in stable condition on oral nonsteroidal anti-inflammatory drugs and colchicine.

At 6-week follow-up she had made a full recovery, and repeat echocardiography demonstrated no recurrence of effusion or evidence of constrictive physiology.

\section{CASE REPORT}

A 63-year-old female presented to hospital with severe, central, sharp chest pain, exacerbated by deep inspiration and upon lying back. She denied cough, fever, or other systemic symptoms. Her past medical history was significant for myelofibrosis, treated with stem cell transplant and complicated by subsequent graft-versushost disease in December 2018.

Baseline blood tests showed normal haemoglobin and total white cell count with mild lymphocytosis and thrombocytopenia. C-reactive protein was elevated at $59 \mathrm{mg} / \mathrm{L}$ (normal range 0-10), as was D-dimer at 743 $\mu \mathrm{g} / \mathrm{L} \quad(0-500)$. High-sensitivity troponin-I levels taken 6 hours apart were in the normal range. A chest radiograph showed a chronically elevated right hemidiaphragm but no acute pathology. Baseline 12-lead ECG showed sinus rhythm with subtle PR-segment depression and saddle ST-segment elevation in the inferolateral leads (Figure 1A). A nasopharyngeal swab, sent for reverse-transcriptase (RT)-PCR assay in line with local policy in place because of the coronavirus disease (COVID-19) pandemic, was negative for all respiratory viruses except severe acute respiratory syndrome coronavirus-2 (SARS-CoV-2). 
A

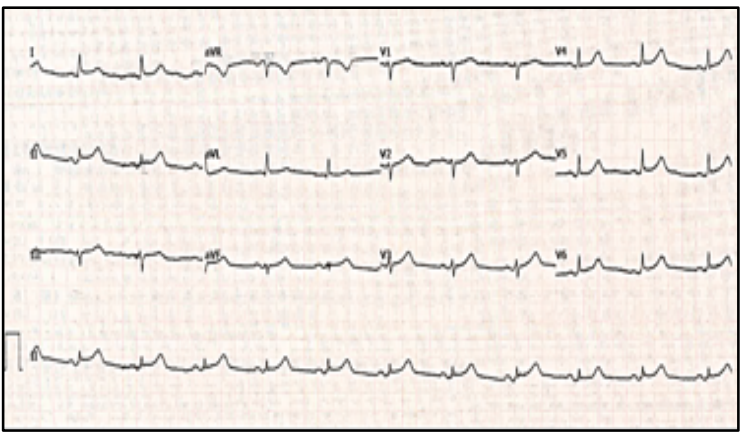

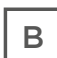

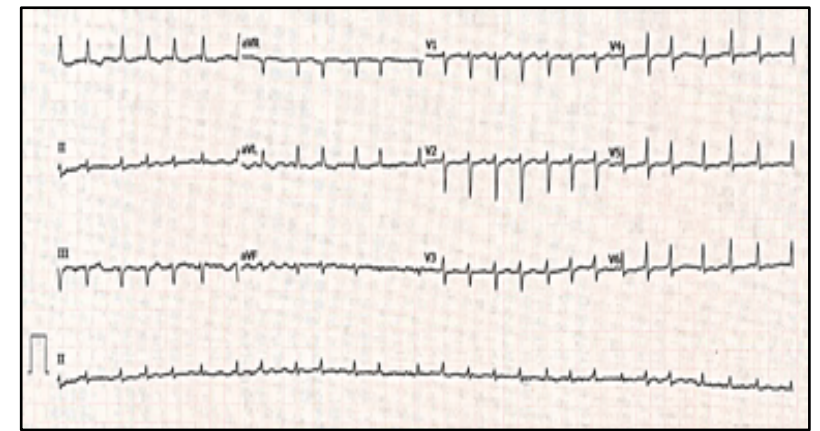

Figure 1: A) Admission ECG demonstrating sinus rhythm with PR depression and a concave ST elevation in inferolateral leads. B) Subsequent ECG showing new-onset atrial fibrillation at a rate of 152 beats per minute with lower voltage complexes in the limb leads and electrical alternans.

Pericarditis was the prime differential diagnosis, but in view of the clinical presentation, raised D-dimer, and history of malignancy, a CT pulmonary angiogram was performed. No pulmonary embolism was seen, but the scan demonstrated collapse and parenchymal inflammatory changes at the right lung base, and minimal pericardial thickening with no pericardial effusion (Figure 2).

The patient was commenced on oral antibiotics for lower respiratory tract infection and nonsteroidal anti-inflammatories (NSAID) for pericarditis.

Four days later she deteriorated, with temperature spikes and rising inflammatory markers. Her ECG at this time showed newonset atrial fibrillation (AF) at a rate of 152 beats per minute, with low-voltage complexes in the limb leads and subtle beat-to-beat alternations of QRS amplitude consistent with electrical alternans (Figure 1B). Her blood pressure had fallen to 108/68; she was becoming pale, diaphoretic, and breathless; and clinical examination indicated elevated central venous pressure. Urgent transthoracic echocardiography (TTE) demonstrated a large global pericardial effusion with a maximum depth of $3.1 \mathrm{~cm}$ and echocardiographic features of tamponade, namely right ventricular diastolic collapse and respiratory variability in mitral valve inflow velocity of $>25 \%$ (Figure 3 ). The left ventricular ejection fraction was well-preserved at 55\%. There were no regional wall motion abnormalities or echo-bright areas of myocardium on TTE suggestive of myocarditis. Given her infective state and her precarious haemodynamic situation, emergency pericardiocentesis was undertaken at the bedside, yielding over $600 \mathrm{~mL}$ of serosanguinous fluid and restoring adequate blood pressure. Analysis of the pericardial fluid showed it to be a sterile, exudative effusion (fluid albumin $28 \mathrm{~g} / \mathrm{L}$ against contemporaneous serum albumin $32 \mathrm{~g} / \mathrm{L}$ ). Cytology revealed heavily bloodstained fluid with occasional macrophages present but no malignant cells. No SARS-CoV-2 or other standard viral RNA was detected in the aspirate.

She made a good recovery thereafter, and was discharged home 48 hours later on NSAID, colchicine $0.5 \mathrm{mg}$ twice per day, bisoprolol $2.5 \mathrm{mg}$ once per day, and a proton-pump inhibitor. AF had resolved following drainage of the fluid and, given that the AF was thought to be triggered by atrial irritation and her CHA2DS2-VASC score was 1 (female sex), an anticoagulant was considered ill-advised.

At 6-week follow-up the patient had made a full symptomatic and functional recovery. Repeat TTE showed good left ventricular systolic function and no recurrence of effusion, nor evidence of pericardial thickening or constrictive physiology.

\section{DISCUSSION}

The authors have presented a case of lone pericarditis as the predominate presenting feature of COVID-19 infection, the most noteworthy feature of which is the rapid progression to cardiac tamponade despite prompt commencement of anti-inflammatory medication. 


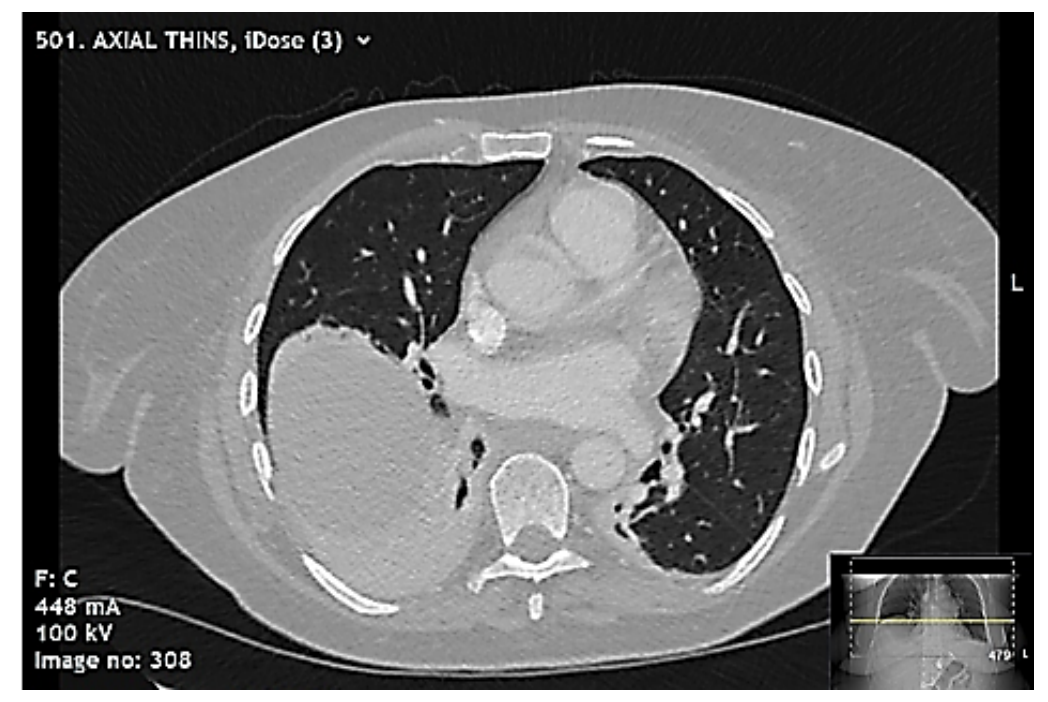

Figure 2: Selected slice from CT pulmonary angiogram demonstrating right lower zone collapse with mild inflammatory changes, mild pericardial thickening, and no pericardial effusion.

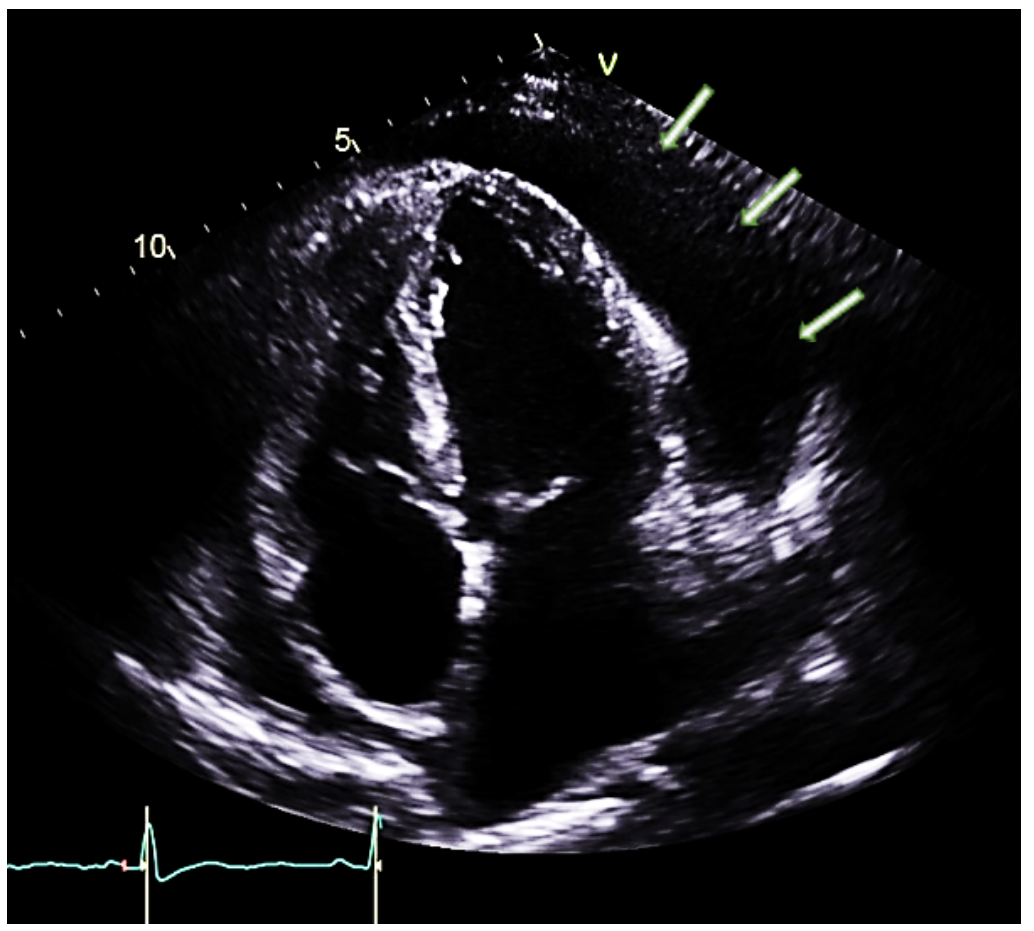

Figure 3: Transthoracic echocardiogram (apical four chamber view) demonstrating large global pericardial effusion (shown with arrows).

Following the first reported cases of COVID-19, caused by SARS-CoV-2, from Wuhan, China in December 2019, the devastating morbidity and mortality of the virus has led to an extraordinary level of global research to better understand the pathophysiology and clinical manifestations of infection. While initial concerns focussed on development of fever, coryzal symptoms, and pneumonia, ${ }^{1}$ appreciation of the extensive range of extrapulmonary symptoms, especially cardiac, has been growing. Despite this, reports of pericardial inflammation and tamponade caused by COVID-19 remain uncommon, particularly in the absence of other systemic features; in many reports myopericarditis complicates COVID-19 pneumonia rather than occurring alone, while 
tamponade, where encountered, tends to develop over a more protracted period than seen in this case. ${ }^{2,3}$ Cardiac involvement, though, does appear to signify a poorer prognosis. ${ }^{4}$

The mechanism of cardiac inflammation in COVID-19 is not well understood at present, though some inferential conclusions have been made based on the pathogenicity of previous coronaviruses, namely SARS-CoV (responsible for the SARS outbreak in 2003) and the Middle East respiratory syndrome-related coronavirus (MERS-CoV). SARS-CoV-2, much like its predecessors, is known to enter myocardial cells through interaction with the angiotensinconverting enzyme-2 receptor. SARS-CoV RNA could be detected in necropsy specimens of human myocardium during the SARS pandemic ${ }^{5}$ and significant macrophage infiltration was also noted, supporting the theory of direct viral invasion and myocardial damage as a mechanism of initial injury. Subsequent stimulation of proinflammatory cytokine production, such as TNFa, IL-6, and IL-8, then occurs. Ultimately, this can be followed by upregulation of interstitial fibrotic pathways, via Smad3-induced modulation of TGFB signalling, ${ }^{6}$ which mediates scarring.

Generally, viral infection is the most common cause of pericarditis. ${ }^{7}$ Previous prospective studies have shown that pericardial effusion is a frequent complication of pericarditis, evident about $73 \%$ of the time, ${ }^{8}$ but is typically mild. Large effusions and tamponade following pericarditis are considerably rarer, occurring in around $2-3 \%$ of cases and generally developing several weeks or months after initial presentation. In the chronic setting, large volumes of fluid (often as much 2-3 litres) can accumulate in the pericardial space without becoming clinically apparent because gradual pericardial stretch offsets any change in pericardial pressure. However, over a shorter timeframe (typically hours to days), the accretion of fluid can quickly outstrip pericardial compliance, thus only a few $\mathrm{mL}$ of exudate may be required for pericardial pressure to exceed right ventricular filling pressure and cause tamponade. ${ }^{9}$ Accumulation of $>600 \mathrm{~mL}$ pericardial fluid over 3-4 days, especially without earlier clinical manifestation, is therefore exceptional.

Whilst viral pericarditis is recognised as the most common aetiological factor, establishing direct causal correlation between viral infection and pericardial inflammation can be challenging. Ideally this would be done by demonstrating viral presence in a pericardial aspirate through culture or RT-PCR testing in a patient with concurrent clinical evidence of systemic infection with the same virus. However, in general clinical practice, as in this case, this is frequently not possible. PierreYves et $a l . .^{10}$ proposed a systematic cascade of investigations to yield a diagnosis in pericarditis, shown in a series of 106 pericardial aspirates over a 7-year period. While a three-fold improvement in diagnostic yield was demonstrated with this method, it is worth noting that PCR gave a positive diagnosis in only 11 out of 37 cases where the diagnosis had not been reached with other methods. Successful detection of SARS-CoV-2 in pericardial fluid using PCR has, to date, been reported exceptionally rarely." The diagnosis is often made based on the temporal association between infection with a biologically plausible aetiological agent and onset of pericarditis, combined with ruling out other potential causes through biochemical, immunological, and microbiological analysis of serum and pericardial fluid.

The patient in this case was started on highdose NSAID and colchicine, as per standard international guidance. ${ }^{12,13}$ The safety of NSAID use during the COVID-19 pandemic had been called into question. Originally, it had been suggested that NSAID use increased cellular expression of the ACE-2 receptor, which could facilitate viral binding and cellular invasion. ${ }^{14}$ However, no clear evidence supporting this assertion has been proposed in the literature. Others had suggested a potential exacerbation of harmful systemic, particularly cardiorenal, effects in people who were unwell and dehydrated due to viral infection, which was compounded by anecdotal reports of individuals who suffered worsening symptoms following administration of ibuprofen. The World Health Organization (WHO) subsequently advised against the use of NSAID because of the potential risk of clinical worsening in patients with SARS-CoV-2 infection. However, the lack of good quality data against NSAID led to a subsequent reversal of that recommendation. Official guidance from the U.S. Food and Drug Administration (FDA) and other international medical bodies now supports NSAID use for those prescribed the drug for 
a pre-existing condition or when no suitable alternative is available. ${ }^{15,16}$ Thus, despite the initial scepticism over the potentially harmful effects of NSAID administration in patients with COVID-19, this remains the recommended treatment for pericarditis.

Colchicine is a well-established primary treatment agent for pericarditis. It exerts an anti-inflammatory effect by inhibiting microtubule formation, which in turn disrupts the nucleotide-binding oligomerisation domainlike receptor pyrin domain-containing-3 (NLRP3) inflammasome. Inhibition of the NLRP3 inflammasome downgrades the release of IL1 , which abrogates the recruitment of myeloid leucocytes, such as neutrophils, monocytes, and macrophages, to the site of injury. ${ }^{17}$ Colchicine may also play a role in diminishing the inflammatory response in the early stages of COVID-19 infection to prevent onset of immunopathological effects including acute respiratory distress syndrome; this is the subject of investigation for several upcoming European trials. ${ }^{18,19}$ For now, colchicine remains a favourable treatment for pericarditis in those concurrently infected with COVID-19.

Corticosteroids are indicated treatment for pericarditis in

as adjunctive patients with incomplete response to NSAID and colchicine, or for those in whom these agents are contraindicated, ${ }^{12}$ but current international guidance advocates avoidance in cases of pericarditis with infective aetiology. However, the beneficial anti-inflammatory properties of corticosteroids may offset the risk of increased susceptibility to viral replication in cases of advanced COVID-19 pneumonia when the immunopathologic response is the predominating issue. $^{20}$ Trial data to clarify the optimal timing and dosage of corticosteroid administration in patients with COVID-19 are eagerly awaited.

\section{CONCLUSION}

This case demonstrates the unusual clinical event of rapid progression from dry pericardium to cardiac tamponade over a few days despite prompt treatment with anti-inflammatory medication. It highlights the potential for patients with COVID-19-related cardiac inflammatory conditions to develop life-threatening effusions within a short timeframe, and the need to be vigilant for this. NSAID and colchicine remain first-line treatments for pericarditis in patients with COVID-19.

\section{References}

1. Guan WJ et al. Clinical characteristics of coronavirus disease 2019 in China. N Engl J Med. 2020;41(22):2130.

2. Hua A et al. Life-threatening cardiac tamponade complicating myopericarditis in COVID-19, Eur Heart J. 2020;DOI:10.1093/eurheartj/ehaa253.

3. Dabbagh MF et al. Cardiac tamponade secondary to COVID-19. JACC Case Rep. 2020;DOI:10.1016/j. jaccas.2020.04.009. [Epub ahead of print]

4. Shi S et al. Association of cardiac injury with mortality in hospitalized patients with COVID-19 in Wuhan, China. JAMA Cardiol. 2020;DOI:10.1001/ jamacardio.2020.0950.

5. Oudit GY et al. SARS-coronavirus modulation of myocardial ACE2 expression and inflammation in patients with SARS. Eur J Clin Invest. 2009;39(7):618-25.

6. Zhao $X$ et al. Severe acute respiratory syndrome-associated coronavirus nucleocap-sid protein interacts with Smad3 and modulates transforming growth factor-beta signaling. J Biol Chem. 2008;283(6):3272-80.

7. Ramasamy $\vee$ et al. Established and novel pathophysiological mechanisms of pericardial injury and constrictive pericarditis. World J Cardiol. 2018;10(9):87-96.

8. Imazio M et al. Good prognosis for pericarditis with and without myocardial involvement: results from a multicenter, prospective cohort study. Circulation. 2013;128(1):42-9

9. Spodick DH. Acute cardiac tamponade. N Engl J Med. 2003;349(7):684-90.

10. Pierre-Yves L et al. Molecular analysis of pericardial fluid: a 7-year experience, Euro Heart J. 2006;27(16):1942-6.

11. Farina A et al. SARS-CoV-2 detection in the pericardial fluid of a patient with cardiac tamponade. Eur J Intern Med. 2020;76:100-1.
12. Adler $Y$ et al. 2015 ESC Guidelines for the diagnosis and management of pericardial diseases: The Task Force for the Diagnosis and Management of Pericardial Diseases of the European Society of Cardiology (ESC) endorsed by: The European Association for Cardio-Thoracic Surgery (EACTS). Eur Heart J. 2015;36(42):2921-64

13. Imazio M, Adler Y. Management of pericardial effusion. Eur Heart J. 2013;34(16):1186-97.

14. Fang $L$ et al. Are patients with hypertension and diabetes mellitus at increased risk for COVID-19 infection? Lancet Respir Med. 2020;8:e21.

15. U.S. Food and Drug Administration (FDA). FDA advises patients on use of non-steroidal anti-inflammatory drugs (NSAIDs) for COVID-19. 2020. Available at: https://www.fda.gov/ drugs/drug-safety-and-availability/ fda-advises-patients-use-nonsteroidal-anti-inflammatory-drugsnsaids-covid-19. Last accessed: 14 December 2020. 
16. World Health Organization (WHO). The use of non-steroidal antiinflammatory drugs (NSAIDs) in patients with COVID-19. 2020 Available at: https://www.who.int/ news-room/commentaries/detail/ the-use-of-non-steroidal-antiinflammatory-drugs-(nsaids)-inpatients-with-covid-19. Last accessed: 14 De-cember 2020
17. Cremer PC et al. Complicated pericarditis: understanding risk factors and patho-physiology to inform imaging and treatment. J Am Coll Cardiol. 2016;68(21):2311-28.

18. Deftereos SG et al. Effect of colchicine vs standard care on cardiac and inflammato-ry biomarkers and clinical outcomes in patients hospitalized with coronavirus dis-ease 2019: the GRECCO-19 randomized clinical trial. JAMA Netw Open. 2020;3(6):e2013136.

19. Schlesinger $\mathrm{N}$ et al. Colchicine in COVID-19: an old drug, new use. Curr Pharmacol Rep. 2020:1-9. [Epub ahead of print].

20. Imazio $M$ et al. Anti-inflammatory therapies for pericardial diseases in the COVID-19 pandemic: safety and potentiality. J Cardiovasc Med. 2020;21(9):625-9. 